\author{
J. R. Fischer ${ }^{1}$ \\ U. Haffner ${ }^{1}$ \\ G. M. Dietrich ${ }^{2}$ \\ B. Spahlinger ${ }^{3}$ \\ D. Geiger ${ }^{1}$ \\ H. Lahm $^{4}$
}

\section{Drittlinientherapie mit Gefitinib beim nicht-kleinzelligen Bronchialkarzinom}

Non-Small-Cell Lung Cancer Third-Line Therapy with Gefitinib

\section{Zusammenfassung}

Mit dem EGFR Tyrosin-Kinase Inhibitor Gefitinib (Iressa ${ }^{\mathrm{TM}}$ ) steht ein neues Therapieprinzip für die Behandlung des nicht-kleinzelligen Bronchialkarzinoms zur Verfügung. Neueste Forschungsarbeiten tragen zu einem besseren Verständnis der Grundlagen der molekularen, zielgerichteten Therapie (Molecular Targeted Therapy, MTT) bei und erlauben es, die bisher vorliegenden Ergebnisse zur klinischen Aktivität von Gefitinib neu zu interpretieren und erstmalig prädiktiv Anhaltspunkte für geeignete Patienten-Subgruppen bei der Therapieentscheidung zu selektionieren.

In der vorliegenden Arbeit werden drei Kasuistiken vorgestellt, an denen aufgezeigt wird, dass vor dem Hintergrund neuer Ergebnisse der translationellen Forschung mit Gefitinib eine wirksame, neue Substanz für die Behandlung chemotherapie-resistenter nicht-kleinzelliger Bronchialkarzinome zur Verfügung steht.

\section{Abstract}

The EGFR-inhibition via tyrosine-kinase-inhibitor gefitinib (Ires$\mathrm{sa}^{\mathrm{TM}}$ ) constitutes a new way to treat non-small-cell lung cancer. Recent research results enable us to better understand the basics of molecular targeted therapy (MTT). These results are helpful to re-interpret the clinical results obtained so far for gefitinib and to consider for the first time in a predictive manner factors for the selection of patients suitable for therapy.

Three case reports are presented in this paper which illustrate that - with view to the results from translational research - the use of gefitinib offers an efficient new therapeutic modality for the treatment of chemotherapy-resistant non-small-cell lung cancer.

\section{Einleitung}

Die eingehende Beschäftigung mit der Pathogenese maligner Erkrankungen hat insbesondere in den letzten zehn Jahren zu einer rasant ansteigenden Anzahl wissenschaftlicher Beiträge zur Identifizierung zellulärer und humoraler Tumormarker geführt, die einerseits wie die Onkogene an der Kanzerogenese maßgeb- lich beteiligt sind und andererseits geeignet erscheinen, als Marker unsere Möglichkeiten zur Diagnose und Prognose insbesondere solider Tumoren entscheidend zu verbessern. Solche molekularen Marker sollten bestenfalls nicht nur Aussagen zum Krankheitsverlauf und zum zu erwartenden Überleben liefern, sondern darüber hinaus auch den behandelnden Arzt bei seiner Therapieentscheidung unterstützen. 
Der epidermale Wachstumsfaktorsrezeptor (EGFR bzw. synonym HER1) gehört zu den HER-Rezeptorproteinen [1]. Die Identifizierung molekularer Targets (Zielgene) und Pathways (Signalketten) innerhalb der zellulär bedeutenden HER-Signalübertragungskaskade hat inzwischen zur Zulassung neuartiger Wirkstoffe geführt, die wesentlich zur onkologisch-therapeutischen Innovation der letzten Jahre beigetragen haben (Abb.1). In vielen humanen soliden Tumoren, darunter das Mamma-, Kolon-, Ovarialkarzinom und NSCLC, wird HER1 stark überexprimiert und ist mit einem schlechten klinischen Verlauf und ungünstiger Überlebensprognose assoziiert [2], wenngleich die Bedeutung dieses prognostischen Faktors für NSCLC als eher gering eingestuft wird [3].

Von Bedeutung sind diese Therapeutika über ihren positiven Beitrag für die Patientenversorgung hinaus dadurch, dass parallel zu ihrer pharmazeutisch-klinischen Entwicklung durch intensive pharmakologische und molekularbiologische Arbeiten wie im Fall von Trastuzumab die in-vivo-Expression des molekularen Targets in Form eines etablierten Tests nachgewiesen werden kann und somit als Tumormarker zur histopathologischen Beurteilung zur Verfügung steht. So ist Trastuzumab (Herceptin ${ }^{\mathrm{TM}}$ ) für Patienten mit einem metastasierten Mammakarzinom empfohlen und zugelassen, deren Tumor HER2 deutlich überexprimiert (HER2 3+). Die Biologie von HER2 unterscheidet sich jedoch deutlich von HER1 - ein spezifischer Ligand konnte bei HER2 bisher nicht identifiziert werden, die Aktivierung der nachgeschalteten Signalkaskade erfolgt durch eine Homodimerisierung beziehungsweise Heterodimerisierungen mit anderen HERRezeptoren. Das Ausmaß der Rezeptorexpression dient im Fall von HER2 als prognostischer und therapeutischer Marker.

Obgleich in verschiedenen soliden Tumoren stark überexprimiert [4], konnte bisher kein gesicherter Zusammenhang zwischen der HER1/EGFR-Überexpression und dem Ansprechen auf eine anti-EGFR-Therapie gezeigt werden. Dies gilt sowohl für den monoklonalen Antikörper Cetuximab (Erbitux ${ }^{\mathrm{TM}}$ ), in diesem Jahr als Inhibitor der extrazellulären HER1/EGFR-Domäne in Europa zur Behandlung des metastasierten kolorektalen Karzinoms zugelassen, wie auch für das Quinazolinderivat Gefitinib (Iressa ${ }^{\mathrm{TM}}$ ), mit dem nun erstmalig ein intrazellulär angreifender Inhibitor der Tyrosin-Kinase-Domäne des EGF-Rezeptors zur Behandlung des metastasierten nicht-kleinzelligen Bronchialkarzinoms zur Verfügung steht [3].

Gefitinib wurde aufgrund seiner in klinischen Phase-II-Studien gezeigten Aktivität und der beeindruckenden Therapieerfolge bei Subgruppen von Patienten als Single-Agent bei der Behandlung des fortgeschrittenen NSCLC bisher in über 20 Ländern einschließlich der USA, Japan, Australien und Kanada zugelassen [5-7]. Mit dem strukturell eng verwandten Erlotinib wurde kürzlich in den USA der zweite Tyrosinkinaseinhibitor zur Rezidivtherapie des fortgeschrittenen Bronchialkarzinoms zugelassen, aufbauend auf überzeugenden Phase-III-Daten, die eine hoch signifikante Überlebenszeitverlängerung aufzeigen konnten [8]. Die Ergebnisse zur Primärtherapie in der Kombination mit Chemotherapie waren für Gefitinib $[9,10]$ und gleichfalls für Erlotinib [11,12] enttäuschend. In keiner der vier großen randomisierten Phase-III-Studien (INTACT-1 und -2, TALENT und TRIBUTE) konnte ein signifikanter Überlebensvorteil für die Gesamtpopulation gezeigt werden.

Diese Ergebnisse haben - auch hinsichtlich der in vivo beeindruckenden additiven Wirkung von Gefitinib in Kombination mit Chemotherapeutika [13] und der fehlenden klinischen Korrelation zwischen Rezeptorstatus und beobachteten Ansprechraten für Gefitinib die Frage aufgeworfen, inwieweit diese TargetedTherapy die in sie gesetzten Hoffnungen erfüllen kann [14]. Zugleich wurde, auch im Hinblick auf die mit Erlotinib erhaltenen Ergebnisse, erkennbar, dass entscheidende Hinweise zur Deutung der klinischen Aktivität durch ein besseres Verständnis der Struktur des pharmakologischen Rezeptors gefunden werden können.

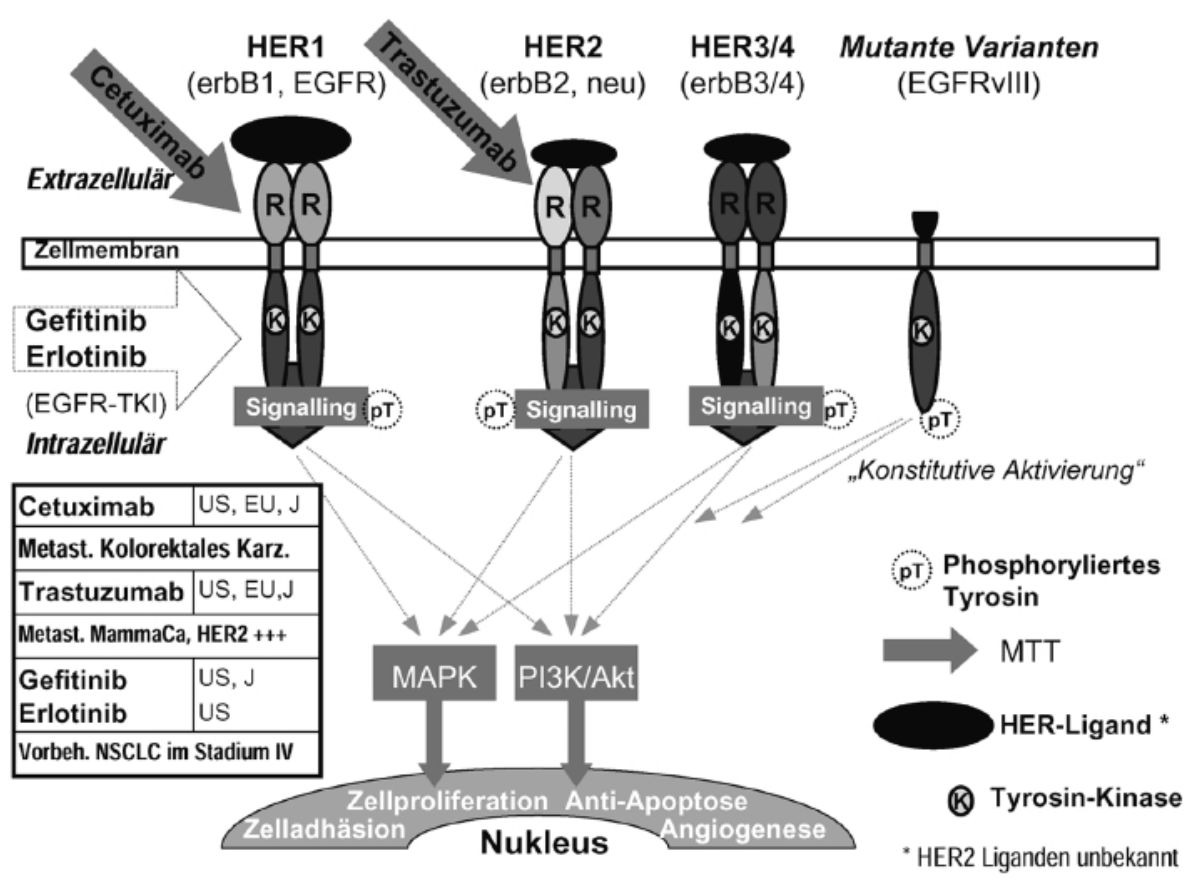

Abb. 1 Neue 'Targeted Drugs', die in den Signalübertragungs-Mechanismus der HER-Familie eingreifen. Im Gegensatz zu beiden monoklonalen Antikörpern Trastuzumab und Cetuximab hemmt Gefitinib intrazellulär die Tyrosin-KinaseDomäne. 
Neben den bekannten vier unterschiedlichen Rezeptortypen (HER1 - HER4), die durch Homo- wie auch Heterodimerisierung eine Aktivierung der nachgeschalteten Signaltransduktionswege ermöglichen, existieren innerhalb der HER-Rezeptorfamilie weitere Rezeptortypen, von denen die 1998 erstmals beschriebene und ausschließlich in verschiedenen Tumorzelltypen gefundene Variante III (EGFRvIII) die bekannteste ist [15].

Zwei neue Arbeiten von Lynch [16] und Paez [17] tragen äußerst bedeutsame Hinweise zu Struktur-Aktivitäts-Beziehungen von Tyrosin-Kinasen bei. Beide Arbeiten aus dem Bereich der translationellen Forschung konnten durch Mutationsanalyse der EGFRkodierenden Sequenz aus dem Tumormaterial von Gefitinib-Respondern durch nachfolgende PCR-Amplifikation und Sequenzierung aufzeigen, dass das EGFR-Gen bei diesen Patienten gehäuft somatische Mutationen in zwei Exons (19 und 21) aufweist, die zu einer Veränderung der ATP- und Gefitinib-Bindungsstelle im Bereich der Tyrosin-Kinase-Domäne des Rezeptorproteins führt. Funktionelle Untersuchungen des exprimierten, veränderten EGFR mit entweder einer In-frame-Deletion (Exon 19) oder einer Punktmutation (Exon 21) zeigten eine erhöhte und länger anhaltende Aktivität nach Stimulation mit EGF sowie eine gesteigerte Sensitivität dieser aberranten EGFR-Formen gegenüber Gefitinib-Inhibition. Folgende Schlussfolgerungen beider Gruppen sind von enormer Bedeutung:

- Die gesteigerte Sensitivität gegenüber Gefitinib trägt zu den klinisch teils sehr ausgeprägten Remissionen bei.

- Der Nachweis dieser EGFR-Mutationen zeigt prädiktiv Gefitinib-Sensitivität auf. Patienten mit Mutationen profitieren demzufolge mehr von einer Behandlung mit Gefitinib als von einer Kombinationschemotherapie.

- Mutationen traten bei bisher 25 bzw. 119 untersuchten NSCLC-Tumorproben in Adenokarzinomen und bronchioalveolären Karzinomen der Lunge häufiger auf als bei anderen Histologien, bei Frauen häufiger auf als bei Männern.

- Ex- und Nichtraucher überwiegen bei Patienten mit ausgeprägten Remissionen [16].

- Die beschriebenen Charakteristika korrelieren mit dem beobachteten Ansprechen in klinischen Studien [7].

Eine nachfolgend veröffentlichte Arbeit der Arbeitsgruppe um Settleman konnte nachweisen, dass Gefitinib-sensitive Tumoren eine potenziell unterschiedliche Signaltransduktion aufzeigen [18]. Das Ansprechen resultiert aus der entstandenen Abhängigkeit der Tumoren vom anti-apoptotischen Akt-Pathway, die EGFR-Inhibierung durch Gefitinib führt zur Aufhebung der das Zellüberleben sichernden Signalübertragung.

Unsere Klinik konnte ca. seit 18 Monaten am Expanded access programm mit Gefitinib teilnehmen. Im Rahmen dieses Compassionate-use-Programms, das den Zeitraum der laufenden Beantragung der europäischen Zulassung abdeckt, konnten wir erste eigene Erfahrungen mit diesem Wirkstoff an über 20 Patienten mit vorbehandelten, metastasierten Bronchialkarzinomen sammeln. Die zuvor beschriebenen Ergebnisse haben uns veranlasst, drei Patienten, die ein signifikantes klinisches Ansprechen aufzeigten, retrospektiv zu analysieren.
Kasuistiken

\section{Fall 1}

Bei Patientin A, einer 59-jährigen Nichtraucherin, wurde radiologisch im August 2002 ein mäßig ausdifferenziertes Adenokarzinom mit geringer Schleimbildung (G2) der rechten Lunge diagnostiziert, nachdem der behandelnde Hausarzt im Rahmen einer Abklärung fibromyalgischer Syndrome einen CEA-Tumormarkeranstieg auf $17 \mathrm{ng} / \mathrm{ml}$ beobachtet hatte. Der Tumor erstreckte sich vom Unter- bis zum Oberlappen und war dort mit der mediastinalen Pleura verwachsen. Weitere Tumorknoten fanden sich in der gesamten rechten Lunge, dem Zwerchfell und der Pleura parietalis. Aufgrund der festgestellten Pleuritis carcinomatosa und der Verwachsung wurde die Erkrankung nach Teilpleurektomie als inoperabel eingestuft (T4N0M1). Vom September 2002 an wurden begleitend Maßnahmen durchgeführt, um den Krankheitsverlauf bestmöglich zu kontrollieren (Tab.1).

Die erneute Progression im Januar 2004 fiel mit einer deutlichen Zunahme der Dyspnoe als wesentlicher krankheitsbedingter Symptomatik zusammen, sodass eine Sauerstofflangzeittherapie erforderlich wurde. Aufgrund der beschränkten BehandlungsOptionen erfolgte im Februar 2004 die Entscheidung zur Einleitung einer Drittlinientherapie mit täglich $250 \mathrm{mg}$ Gefitinib. Die Patientin berichtete innerhalb der ersten Behandlungswochen über eine deutliche Abnahme der belastenden Dyspnoe, gleichzeitig fiel der CEA-Wert von 15,0 ng/ml innerhalb von $10 \mathrm{Be}-$ handlungswochen auf $5,8 \mathrm{ng} / \mathrm{ml}$ ab, einem Werte nahe des Normalbereiches für Nichtraucher (zu 99\% $<5.0 \mathrm{ng} / \mathrm{ml}$ ) ab.

Die radiologischen Kontrollen unterstrichen das klinische Bild einer nahezu kompletten Remission (Abb. 2). Als therapiebekannte Nebenwirkungen traten leichte Diarrhöen und Erbrechen innerhalb der ersten zwei Behandlungswochen sowie Pusteln im Bereich der Nase auf. Bei verbesserter Lebensqualität stabilisierte sich die beobachtete Remission mit weiterer Rückbildungstendenz über den Sommer 2004, sodass die Therapie bisher fortgeführt werden konnte.

Tab. 1 Übersicht zu den durchgeführten therapeutischen Maßnahmen bei Patientin $A$

\begin{tabular}{|c|c|}
\hline August 2002 & $\begin{array}{l}\text { - Thorakotomie rechts mit atypischer Tumorresektion } \\
\text { aus ML und UL, PE Zwerchfell und Teilpleurektomie }\end{array}$ \\
\hline September 2002 & $\begin{array}{l}\text { - } 3 \text { Zyklen Chemotherapie mit Gemcitabin und Carbo- } \\
\text { platin }\end{array}$ \\
\hline November 2002 & $\begin{array}{l}\text { - Einleitung } 2^{\text {nd }} \text {-line Therapie mit Docetaxel }(q 3 w \times 6) \\
\text { nach Progress }\end{array}$ \\
\hline Mai 2003 & $\begin{array}{l}\text { - } 6 \text { Wochen nach Absetzen } 2^{\text {nd-line erneute retro- }} \\
\text { sternale Progression, nachfolgend palliative Radiatio } \\
\text { des Bereichs mit } 33 \text { Gy Gesamtdosis }\end{array}$ \\
\hline Januar 2004 & $\begin{array}{l}\text { - erneute pulmonale Progression, Indikation zu } 3^{\text {rd }} \text {-line } \\
\text { Therapie }\end{array}$ \\
\hline Februar 2004 & $\begin{array}{l}\text { - Beginn der EGFR-TKI-Therapie mit Gefitinib } \\
\text { (250 mg o.d.) }\end{array}$ \\
\hline September 2004 & - Fortführung der anti-EGFR-Therapie mit Gefitinib \\
\hline
\end{tabular}




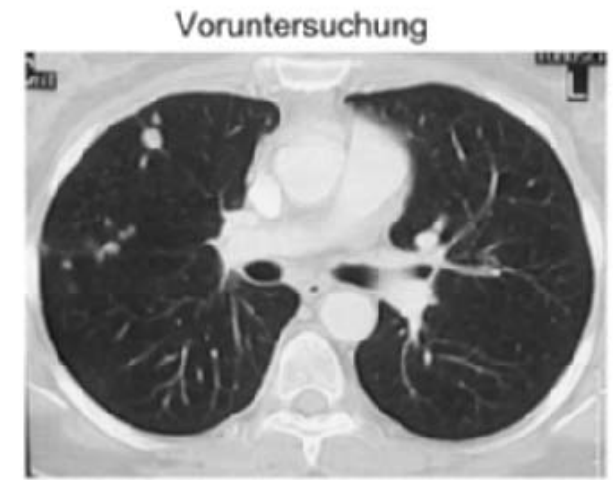

Helicoidales Computertomogramm,

$5 \mathrm{~mm}$ Schichtdicke, Kontrastmittel
Therapiekontrolle Woche 12

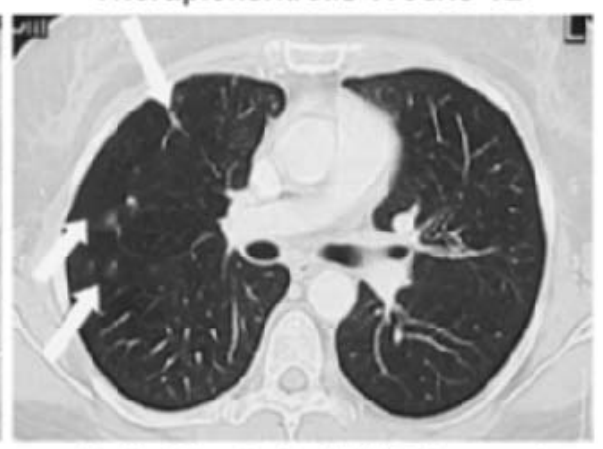

Verlaufskontrolle KW 12, Parameter

für CT identisch zu Voruntersuchung
Abb. 2 Vergleich der Vorher-NachherAufnahmen der Patientin A im CT während des initialen dreimonatigen Behandlungszeitraums.

\section{Fall 2}

Diese 79-jährige Patientin hatte im Januar 2003 ihren behandelnden Arzt konsultiert aufgrund von Schmerzen im Bereich des Brustkorbes. Diagnostisch ließ sich ein großer rechtsseitiger Pleuraerguss nachweisen. Röntgenologisch zeigte sich eine vermehrte Lungenzeichnung wie bei einer Lymphangiosis Carcinomatosa üblich, mehrere parenchymale Knoten in beiden Lungen waren erkennbar. Die TNM-Klassifikation nach der 5. Auflage von 1997 [19] erfolgte als cT2cNXM1. Histologisch wurde ein Adenokarzinom bestimmt. Nachfolgend wurde videoendoskopisch eine rechtsseitige Teilpleurektomie zwecks Pleurodese durchgeführt. In Betracht des Alters der Patientin wurde die Primärtherapie vom Frühjahr 2003 an mit Gemcitabin mono durchgeführt. Eine erneute Tumorprogression im Herbst 2003 einbezüglich pulmonaler Lymphangiosis veranlasste zur Einleitung der Zweitlinientherapie mit Docetaxel, insgesamt drei Zyklen wurden von Dezember 2003 bis Februar 2004 appliziert.

Aufgrund des sich verschlechternden Performance Status (ECOG 4) und Zeichen zunehmender Komorbidität (Grad IV Asthenie und Kachexie) wurde die Therapie schlussendlich gestoppt. Der CEA-Wert stieg zugleich innerhalb von wenigen Wochen deutlich an (20,3 auf 29,4 ng/ml), die spirometrischen Untersuchungen zeigten eine zunehmend schwere und vorwiegend restriktive Ventilationsstörung auf $\left(R_{\text {tot }} 0.5 \mathrm{kPA}\right.$ *s/l, Tiffeneau 99,5\%, FEV 0,79 l, ITGV 52,8\%, TLC 42,9\%).

Die Angehörigen der Patientin setzten sich in starkem Maße für weitere therapeutische Maßnahmen ein, für die aufgrund der vorangegangenen Therapien und Vorerkrankungen (Aortenklappen- und Mitralinsuffizienz) wenig Alternativen zur Auswahl standen. Die Verordnung von Gefitinib (250 mg/dies p.o.) vom Februar 2004 an führte zu einer außerordentlichen Verbesserung des Allgemeinzustandes (ECOG 1) bei gleichzeitiger Halbierung des CEA-Wertes, einem Anstieg der Sauerstoffsättigung von 80 auf $93-94 \%$ und einer bildgebend klar erkennbaren Remission (Abb. 3). Gelegentliche Übelkeit (NCI-CTC Grad 1-2) wurde als einzige Therapie-induzierte Nebenwirkung berichtet.
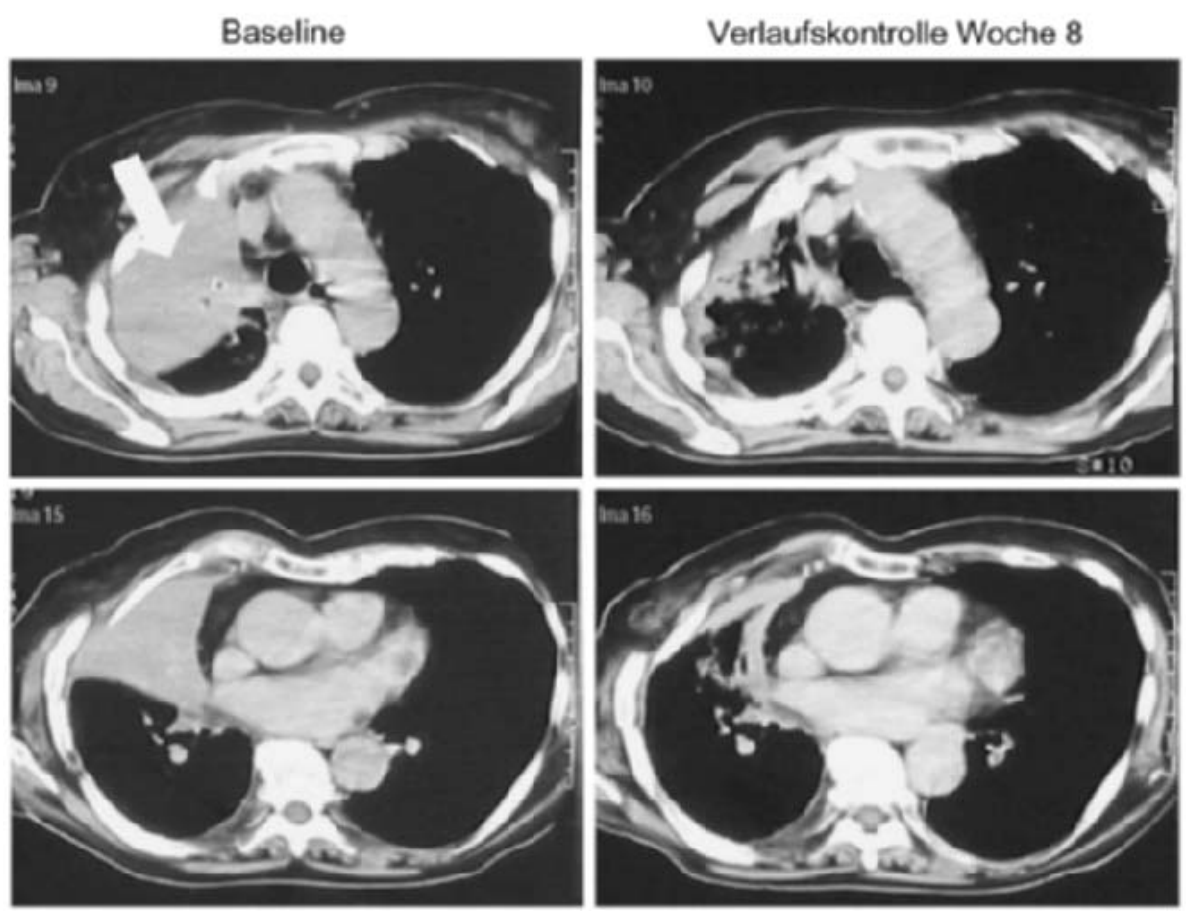

Abb. 3 Darstellung zweier Schnitte aus dem CT der Patientin C vom Oktober 2003 (nach Primärtherapie) und nach 8-wöchiger EGFR-TKI-Therapie. Zu Beginn der Drittlinientherapie wurden aufgrund des schlechten Allgemeinzustandes der Patientin keine gesonderten Aufnahmen angefertigt. 


\section{Fall 3}

Im September 2003 wurde bei einer 47-jährigen ehemaligen Raucherin, die zur diagnostischen Abklärung von Schmerzen in Brustwand und Nacken, einem trockenen, anhaltenden Husten und Belastungsdyspnoe hospitalisiert worden war, ein linksseitiges Adenokarzinom festgestellt. Im Rahmen der weiteren Untersuchungen wurden neben dem in Ober-, Unterlappen und Lingula infiltrierenden Primärtumor multiple, beidseitige Läsionen gefunden sowie Metastasen in Leber und Nebennieren (T4N2M1). Aufgrund der umfassenden Metastasierung wurde sofort mit einer systemischen Chemotherapie begonnen. Die Erst- und Zweitlinientherapie sowie begleitende und radiotherapeutische Maßnahmen führten nur zu kurzzeitigen bzw. keinen Behandlungserfolgen, wie das Auftreten weiterer metastatischer Absiedlungen in Knochen und in beiden Großhirnhemisphären zeigte (Abb.4). Anfang April 2004 wurde die Chemotherapie nach Infektion und resistierendem Fieber trotz kurzfristiger i.v. Antibiotika-Behandlung (Abbruch aufgrund von Diarrhöen), Gewichtsverlust (NCI-CTC Grad 2), Tumoranämie (Gabe von Ery- throzytenkonzentraten) und abfallendem Karnofsky-Index abgesetzt.

Die nachfolgend begonnene Gefitinib-Therapie mit der Standarddosis von $250 \mathrm{mg}$ täglich musste nach wenigen Tagen unterbrochen werden, da moderate Diarrhöen, insbesondere aber starke Bauchkrämpfe auftraten, die jedoch mit Hilfe von Buscopan beseitigt werden konnten. Nach Wiederaufnahme bei gleichzeitigem Ausschleichen von Kortison wurde die anti-EGFRTherapie besser vertragen. Die erste Verlaufskontrolle nach vier Wochen mittels Röntgenthorax zeigte eine eindrucksvolle Rückbildung der massiven beidseitigen Lungenmetastasierung (Abb.5), sodass die Therapie seitdem fortgesetzt wurde. Die erzielte Remission stellte sich im September 2004, fünf Monate nach Therapiebeginn, weiterhin als stabil dar. Die im Zusammenhang mit der Lebermetastasierung zu sehenden initial pathologisch veränderten Leberwerte ( II Toxizität $\gamma$-GT und AP) haben sich unter Therapie weitgehend normalisiert.

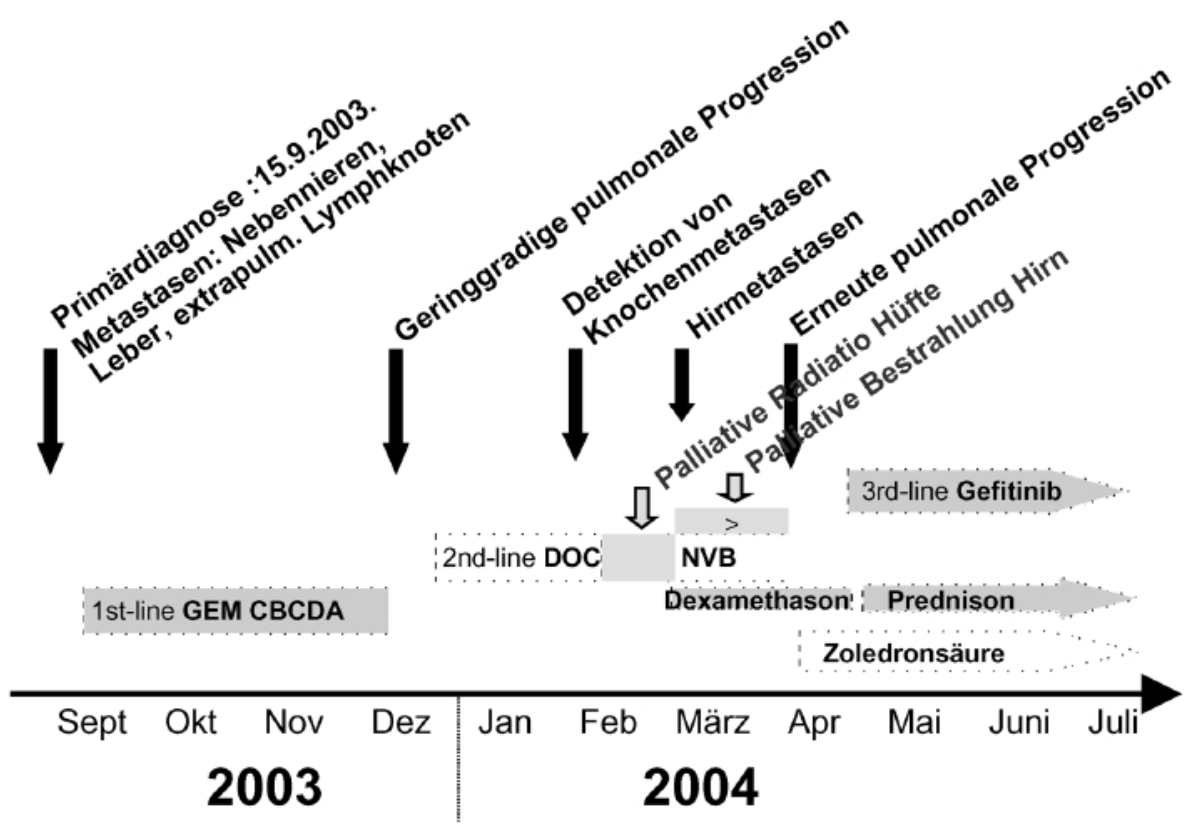

Thorax Baseline (16 Apr 04)

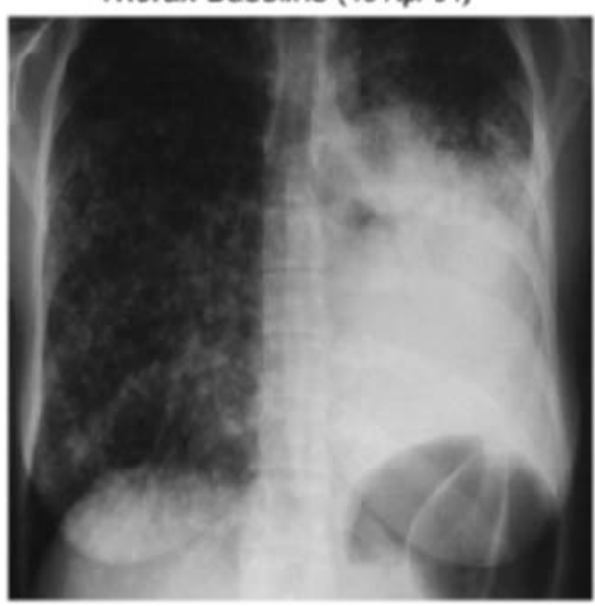

Abb. 4 Zusammenfassende Darstellung des klinischen Verlaufs 2003-04 sowie durchgeführter Maßnahmen bei Patientin C.
Abb. 5 Röntgen-Thoraxaufnahme der 47-jährigen Patientin C. In der rechts dargestellten Verlaufskontrolle ist die schnelle Rückbildung der massiven multiplen beidseitigen pulmonalen Metastasierung gut zu erkennen. 
15/70 Adenokarzinomen (21\%) wiesen EGFR-TK-Mutationen auf, in anderen Histologien konnte nur eine Mutation $(2 \% ; 1 / 49)$

Alle drei Patientinnen sind vortherapiert mit mindestens einem Progress unter Therapie, weisen Adenokarzinome auf und sind Nicht- $(2 / 3)$ bzw. Ex-Raucher. Eine Nachbetrachtung der morphologischen Befunde zeigte, dass in allen drei Fällen eine eindeutige Zuordnung zum Adenotyp als führender histologischer Ausprägung möglich war. Alle drei Patientinnen stehen weiterhin unter Behandlung, aus diesem Grund sind Aussagen zur progressionsfreien Zeit noch verfrüht. CEA erwies sich bei zwei der Patientinnen als geeigneter Marker zur Verlaufskontrolle in Übereinstimmung mit den klinischen und radiologischen Befunden. Eine Erklärung des fulminanten klinischen Ansprechens bei diesen drei Patientinnen ist aufgrund der vorliegenden immunochemischen, histologischen und klinischen Fakten vordergründig soweit nicht möglich.

Festzuhalten ist aber in jedem Fall, dass die Rate der unspezifischen Nebenwirkungen - unter Berücksichtigung der Beobachtungen in über 20 Patienten - im Vergleich zu etablierten Chemotherapieregimen bemerkenswert gering ist. Unter den drei beschriebenen Patientinnen ist ein einziges Ereignis (spastische Bauchkrämpfe) zu vermerken, das zu einer Unterbrechung der Therapie führte. Bei den Blutwerten und der Klinischen Chemie setzt sich diese Beobachtung fort, Abweichungen vom Normalbereich sind in erster Linie durch die Erkrankung bedingt.

\section{Diskussion}

Die Ergebnisse der intensiven Forschung der letzten Jahre zur molekularen Biologie des HER-Rezeptors helfen weiter, das Wirkprinzip dieser Molecular Targeted Therapy (MTT) inzwischen in Ansätzen zu verstehen und im Fall von Gefitinib erste Voraussagen zur Selektion, zur Prognose und zum Ansprechen von NSCLC-Patienten zu erhalten. Vor diesem Hintergrund ist eine retrospektive Neubewertung vorliegender Daten zur klinischen Aktivität von Gefitinib geboten. Erst das Verständnis des Zusammenspiels aus Rezeptorhäufigkeit in normalen und malignen Zellen, Regulation der Rezeptorexpression, Rezeptoraktivierung oder -inhibition sowie der Identifizierung von somatischen Rezeptormutationen erlaubt es, den therapeutischen Wert und das Einsatzgebiet dieser Therapie zu bestimmen. Hierzu gehört auch die Bewertung histopathologischer Unterschiede in Bezug auf den Rezeptorstatus.

Die Aussagen der beiden Bostoner Arbeitsgruppen sind eindeutig: Lynch [16] konnte in 8 von 9 zugänglichen Histologien signifikanter Responder, bei denen ein Ansprechen zwischen 4,3 und 33,5 Monaten beobachtet wurde, Mutationen der Tyrosin-Kinase-Domäne feststellen. In einer entsprechenden Vergleichsgruppe von Gefitinib-refraktären Patienten wurde keine einzige $\mathrm{Mu}$ tation festgestellt ( $p<0.001$ in Fischer's two-sided exact test). Ähnliche Ergebnisse berichtet Paez [17] mit 5/5 Mutationen bei Respondern versus 0/4 bei Nichtrespondern ( $p<0.003$ ). Alle Mutationen wurden in Adenokarzinomen zuzüglich Bronchioalveolären Karzinomen (BAK) nachgewiesen und stammten zu einem hohen Anteil von nichtrauchenden Frauen. Als weitere Kontrolle wurden Histologien von 119 NSCLC-Patienten aus Japan und den USA untersucht, die keine Gefitinib-Behandlung erhalten hatten: nachgewiesen werden; Mutationen waren ausgeprägter bei Frauen (20\%; 9/45) als bei Männern (9\%; 7/74) zu finden. Die Ergebnisse beider Gruppen stimmen zudem überein mit den Aussagen von Miller [20], der retrospektiv die Daten von 139 im Memorial Sloan-Kettering Cancer Center mit Gefitinib behandelten Patienten auf prädiktive Faktoren überprüft hat. Das Vorhandensein eines Adenokarzinoms vom bronchioalveolären Subtyp und Nikotinkarenz konnten als unabhängige prädiktive Faktoren für das Ansprechen auf Gefitinib bestätigt werden. Miller und Pao konnten in einer weiteren Arbeit aufzeigen, dass EGFR-Mutationen in Adenokarzinomen von chemonaiven Nichtrauchern im Gegensatz zu Adenokarzinomen nicht vorbehandelter Raucher in signifikant höherem Maße auftreten [21]; die Adenokarzinome von Nichtrauchern werden von den Autoren demzufolge als eine neue „distinkte Subgruppe“ von Bronchialkarzinomen beschrieben.

Bei dem als Meilenstein in der Behandlung der ChronischenMyeloischen Leukämie angesehenen Imatinib (Glivec ${ }^{\mathrm{TM}}$ ), das den BCR-ABL-Signalweg in Philadelphia-Chromosom-positiven Patienten inhibiert, konnte das Konzept der Inhibition von Tyrosin-Kinasen inzwischen gleichfalls bei komplexen, soliden Tumoren bestätigt werden. Imatinib wirkt bei metastasierten gastrointestinalen Stromatumoren (GIST) über die Inhibition von KIT-Mutationen im Bereich der Tyrosin-Kinase-Domäne und führt bei solch CD117-positiven Patienten zu exzellenten Behandlungsergebnissen. Die bisherigen Ergebnisse beider Substanzen, Imatinib bei GIST und Gefitinib bei Adenokarzinomen der Lunge, unterstreichen die Eignung der Tyrosine-Kinase-Inhibition als onkologisches Therapieprinzip.

Vor diesem Hintergrund ist das Fazit zu ziehen, dass nach unserer eigenen Erfahrung bei der Drittlinientherapie mit Gefitinib bei einzelnen Patienten beeindruckende und mitteilenswerte Behandlungserfolge erzielt werden können. Leider stehen uns zur Zeit für die klinische Routine EGFR-TK-Mutationstests noch nicht zur Verfügung, an denen wir Behandlungserfolge überprüfen bzw. Patienten vor Therapiebeginn auf Ihre genotypische Eignung überprüfen können. Es ist zudem davon auszugehen, dass auch chemotherapeutisch nicht-vorbehandelte Patienten von dieser Therapieform profitieren, unter Umständen sogar in noch stärkerem Maße. Die Beobachtung signifikanter Therapieerfolge innerhalb des eigenen Patientengutes sollte insbesondere beim Einsatz neuer Therapien Ansporn sein, Responder kasuistisch-retrospektiv einer genauen Überprüfung zu unterziehen - wie durch diese Arbeit dargestellt - um in Übereinstimmung mit neuen Ergebnissen der translationellen Forschung im therapeutischen Alltag neue oder adaptierte Therapieentscheidungen $\mathrm{zu}$ treffen. 
${ }^{1}$ Grünwald W, Hidalgo M. Developing Inhibitors of the Epidermal Growth Factor Receptor for Cancer Treatment. J Natl Canc Inst 2003; 95: $851-867$

2 Janmaat ML, Giaccone G. Small-Molecule Epidermal Growth Factor Receptor Tyrosine Kinase Inhibitors. The Oncologist 2003; 8: 576-586

${ }^{3}$ Meert AP, Martin B, Delmotte P et al. The role of EGF-R expression on patient survival in lung cancer: a systematic review with meta-analysis. Eur Respir J 2002; 4: 975 - 981

${ }^{4}$ Salomon D, Brandt R, Ciardello F et al. Epidermal Growth Factor-Related Peptides and their Receptors in Human Malignancies. Crit Rev Oncol Hematol 1995; 19: $183-232$

${ }^{5}$ Cohen MH, Williams GA, Sridhara R et al. United States Food and Drug Administration Drug Approval summary: Gefitinib (ZD 1839; Iressa) tablets. Clin Cancer Res 2004; 15: 1212 - 1218

${ }^{6}$ Fukuoka M, Yano S, Giaccone G et al. Multi-institutional randomized phase II trial of gefitinib for previously treated patients with advanced non-small-cell lung cancer. J Clin Oncol 2003; 21: 2237-2246

${ }^{7}$ Kris MG, Natale RB, Herbst RS et al. Efficacy of gefitinib, an inhibitor of the epidermal growth factor receptor tyrosine kinase, in symptomatic patients with non-small cell lung cancer. A randomised trial. JAMA 2003; 290: $149-158$

${ }^{8}$ Shepherd FA, Pereira J, Ciuleanu TE et al. A randomized placebo-controlled trial of Erlotinib in patients with advanced non-small cell lung cancer (NSCLC) following failure of $1^{\text {st }}$ line or $2^{\text {nd }}$ line chemotherapy. A National Cancer Institute of Canada Clinical Trials Group (NCIC CTG) trial. Proc ASCO 2004; 23: Abst. 7022

${ }^{9}$ Giaccone G, Herbst RS, Manegold C et al. Gefitinib in combination with gemcitabine and cisplatin in advanced non-small-cell lung cancer: A phase III trial - INTACT 1. J Clin Oncol 2004; 22: 777-784

${ }^{10}$ Herbst RS, Giaccone G, Schiller JH et al. Gefitinib in combination with paclitaxel and carboplatin in advanced non-small-cell lung cancer: A Phase III Trial: INTACT 2. J Clin Oncol 2004; 22: 785-794

${ }^{11}$ Gatzemeier U, Pluzanska A, Szeczesna E et al. Results of a Phase III Trial of Erlotinib (OSI-774) combined with Cisplatin and Gemcitabine Chemotherapy in advanced non-small-cell lung cancer (NSCLC). Proc Am Soc Clin Oncol 2004; 23: Abst. 7010

${ }^{12}$ Herbst RS, Prager D, Hermann R et al. TRIBUTE - A phase III trial of erlotinib (OSI-774) combined with carboplatin and paclitaxel (CP) chemotherapy in advanced non-small cell lung cancer (NSCLC). Proc Am Soc Clin Oncol 2004; 23: Abst. 7011

${ }^{13}$ Ciardello F, Caputo R, Bianco R et al. Antitumor Effect and Potentiation of Cytotoxic drugs Activity in Human Cancer Cells by ZD-1839 (Iressa), an Epidermal Growth Factor Receptor-Selective Tyrosine Kinase Inhibitor. Clin Cancer Res 2000; 6: 2053 - 2063

${ }^{14}$ Baselga J. Combining the Anti-EGFR Agent Gefitinib with Chemotherapy in Non-Small-Cell Lung Cancer: How Do We Go From INTACT to Impact? J Clin Oncol 2004; 22: 759-761

${ }^{15}$ Moscatello DK, Holgado-Madruga M, Emlet DR et al. Constitutive activation of phosphatidylinositol 3-kinase by a naturally occurring mutant epidermal growth factor receptor. J Biol Chem 1998; 273: 200-206

${ }^{16}$ Lynch TJ, Bell DW, Sordella R et al. Activating Mutations in the Epidermal Growth Factor Receptor Underlying Responsiveness of NonSmall-Cell Lung Cancer to Gefitinib. N Engl J Med 2004; 350: $2129-2139$

${ }^{17}$ Paez JG, Jänne PA, Lee GC et al. EGFR Mutations in Lung Cancer. Correlation with Clinical Response to Gefitinib Therapy. Science 2004; 304: $1497-1500$

18 Sordella R, Bell DW, Haber DA et al. Gefitinib-Sensitizing EGFR Mutations in Lung Cancer Activate Anti-Apoptotic Pathways. Science 2004; 305: $1163-1167$

${ }^{19}$ Wittekind C, Wagner G (Hrsg). TNM-Klassifikation maligner Tumoren, 5. Auflage. Berlin Heidelberg: Springer, 1997: 91

${ }^{20}$ Miller VA, Kris MG, Shah N et al. Bronchioalveolar Pathologic Subtype and Smoking Predict Sensitivity to Gefitinib in Advanced Non-SmallCell Lung Cancer. J Clin Oncol 2004; 22: 1103 - 1109

${ }^{21}$ Pao W, Miller V, Zakowski $M$ et al. EGF receptor gene mutations are common in lung cancers from "never smokers“ and are associated with sensitivity of tumors to gefitinib and erlotinib. Proc Natl Acad Sci 2004; 101: 13306-13311

\section{Die Geschichte der" „Selkundenluft"}

Journalistenwettbewerb zum Thema „COPD“:

Aus „FEV “ wird „Sekundenluft“

\section{Auf der Suche nach Verständnis}

Einprägsame Begriffe und Übersetzungen für medizinische Fachbegriffe tragen maßgeblich zur breiten Aufklärung über ein Krankheitsbild bei, vor allem bei noch weitgehend unbekannten und unterschätzten Erkrankungen wie der COPD. Um die breite Öffentlichkeit besser aufklären zu können, suchte deshalb der Deutsche Lungentag e.V. gemeinsam mit Journalisten eine allgemeinverständliche Übersetzung des Lungenfunktionsparameters „, $\mathrm{FEV}_{1}$ “. Aus zahlreichen Einsendungen ging der Begriff „Sekundenluft“ als Sieger hervor. Dieser Begriff soll es in Zukunft den Patienten erleichtern, ihren Arzt auf eine Prüfung der Lungenfunktion anzusprechen.

\section{Ist der Begriff Sekundenluft praxistauglich?}

Während des Kongresses der Deutschen Gesellschaft für Pneumologie 2005 in Berlin wurde eine Umfrage unter den teilnehmenden Pneumologen durchgeführt. Wir wollten wissen, ob Sekundenluft als Synonym für $\mathrm{FEV}_{1}$ im Praxisalltag Chancen hat.

\section{Hier die Ergebnisse der Befragung:}

Auf unsere Fragen haben 61 Kolleginnen und Kollegen geantwortet. $54 \%$ der Befragten finden, dass der Begriff „Sekundenluft“ praxisorientiert und allgemeinverständlich (41\%) ist. 56\% halten Sekundenluft für eine zutreffende Übersetzung der $\mathrm{FEV}_{1}$. Die Erwartungen, dass der Begriff in der Praxis (Erklärungs-)Zeit spart sind zurückhaltend (28\% Zustimmung). Auch sind sich viele Pneumologen noch unschlüssig, ob sie den Begriff in der täglichen Arbeit verwenden werden (39\% Zustimmung).

Wir bedanken uns bei allen, die an der Umfrage teilgenommen haben. 